\title{
Using the mathematical modeling approach to access the behavior of plant antioxidant system under abiotic stress conditions
}

\author{
A. Bobrovskikh ${ }^{1,2 *}$, A. Doroshkov ${ }^{1,2}$ \\ ${ }^{1}$ Institute of Cytology and Genetics SB RAS, Novosibirsk, Russia \\ ${ }^{2}$ Novosibirsk State University, Novosibirsk, Russia \\ *e-mail:avb@bionet.nsc.ru
}

Key words: antioxidant system, modelling, ROS, stress response

Motivation and Aim: Reactive oxygen species (ROS) is one of the key factors that damages living systems. ROS production during stress dramatically increases, which causes membrane damage and can lead to death of the living system. The plants have an antioxidant system (AOS) that effectively fights against the ROS. Biochemistry of this system is well-known. AOS includes a number of antioxidant enzymes (ascorbateglutathione cycle, catalase, superoxide dismutase, catalase) and antioxidant species (ascorbate, glutathione). However, the regulation of this system insufficiently studied. There are many kinetic data on antioxidants species and expression data. Integration of this data and dynamic modeling is a perspective approach to describing the complex response of the system to external disturbances, such as abiotic stress.

Methods and Algorithms: Literature sources were used to obtain kinetic data. Expression experiments was extracted from GEO databases. We used the COPASI software [1] to build a dynamic model of the antioxidant system.

Results: We present a new model of AOS in a plant cell. In addition to chloroplasts, models of peroxisome and mitochondrial components of the AOS were created. Based on these data, stationary states are calculated in normal and stressed conditions (salt stress, water-deficient).

Conclusion: Our study includes a complex description of the dynamics of the plant's AOS in response to stress, taking into account the division into compartments. The kinetic mechanisms and kinetic parameters of enzymes of the antioxidant system, concentration of enzymes in different cell compartments and generation rates of free radicals are based on the literature data and databases.

Acknowledgements: We thank Dr. Alexey Kolodkin for consultations that improved the model quality.

\section{References}

1. Hoops S. et al. (2006) COPASI — a complex pathway simulator. Bioinformatics. 22(24):3067-3074. 